\title{
Council Report
}

\section{Benzodiazepines: risks, benefits or dependence. A re-evaluation CR59. 1997. 10 pp. $\$ 5.00$}

This Council Report from the College emphasises that there is still a role for the prescribing of benzodiazepines for anxiety, insomnia and some forms of epilepsy and spasticity. The significant risk of dependence in patients treated for longer than one month must, however, be taken into consideration by all doctors. The report recommends that every clinician should examine the benefit-risk ratio in each individual case early in treatment, so that the risks of dependence can be balanced against the benefits of treatment.

Since the publication of the College's original statement on benzodiazepine dependency in 1988, there have been further developments in the general understanding of the effects of longterm prescribing of this class of drugs. The report acknowledges that short-term prescribing of benzodiazepines is often appropriate for the relief of anxiety and insomnia. There are also occasions when long-term prescribing is justified because of the absence of alternative effective treatments or because such prescribing may reduce the risk of more serious problems (e.g. alcohol dependence). Long-term prescribing should, however, never be the first-choice treatment.

The report reviews the benefits and risks of prescribing benzodiazepines. The main benefits are the reduction of anxiety and the short-term treatment of insomnia. However, these drugs also reduce anxiety in people who are depressed; act as anticonvulsants and muscle relaxants; and are sometimes effective in the treatment of excitement, agitation and severe psychotic states. Their risks include: masking the symptoms of depression, misuse, cognitive and psychomotor impairment, tolerance, and disinhibition.
The report also reminds doctors that:

(a) depression is not an indication for benzodiazepines and that they may precipitate suicide attempts or aggressive behaviour in impulsive individuals;

(b) as a small but important sector of the population misuses benzodiazepines they should always remember that the medication they prescribe may fall into the wrong hands;

(c) cognitive impairment (such as memory disturbance) and psychomotor impairment may be important side-effects of benzodiazepines;

(d) the combination of benzodiazepines and alcohol is both common and dangerous and may exacerbate aggressive behaviour;

(e) the pharmaceutical industry should consider producing formulations of their products at lower strengths which could be used to help patients reduce their excessive doses.

"The advice given in the College's earlier report has been acted on and now there are relatively few patients who take benzodiazepines long term," stressed Dr R. E. Kendell. President of the College (Royal College of Psychiatrists, 1988). "Whilst such prescribing can sometimes be justified in an individual case, it should always be questioned by the clinician and alternative ways of treating the patient kept under constant review."

\section{Reference}

Royal College of PSychiatrists (1988) Benzodiazepines and dependence: a College statement. Psychiatric Bulletin, 12, 107-108.

For further information contact External Affairs Department on 01712352351 ext. 127 or 154 . 